\title{
The Formation of Protein Structure
}

\author{
J. Bohr, H.G. Bohr, S. Brunak \\ The Technical University of Denmark
}

Dynamically induced curvature owing to long-range excitations along the backbones of protein molecules with non-linear elastic properties may control the folding of proteins.

Several of the more amazing phenomena in living biological systems concern their ability to form structure. At the macroscopic level it is the ability to form shape - the process of morphogenesis. On the cellular scale it is the capacity to selforganize and assemble molecules to form larger aggregated structures. On the molecular scale it is the ability of chain molecules such as DNA and proteins to acquire specific three-dimensional structures - an ability which to our knowledge is only utilized in biological systems so far.

Proteins form reproducible structures, i.e., chemically identical proteins acquire identical three-dimensional structures. This may not seem surprising at first. However, it is a unique characteristic as other long polymers do not possess this ability. The process whereby proteins acquire their reproducible structures is called protein folding. Protein folding the formation of structure in proteins - is of fundamental importance in biological systems because the ability of a protein to perform a function - to act as an enzyme, for instance - is fundamentally connected with the structure of the protein. The mis-

Jakob Bohr has been a Research Professor of Physics in the Department of Physics, The Technical University of Denmark (DTU), DK-2800 Lyngby, since 1994. He studied civil engineering at the DTU before switching to research on diffraction studies for which he received his $\mathrm{PhD}$ from the DTU in 1984. He joined the Risø National Laboratory in 1983 and became Research Professor in 1991 having spent two years working in the USA.

Henrik Bohr became an Associate Professor in the Centre for Biological Sequences Analyses, part of the DTU's Physical Chemistry Department, in 1994. Professor Bohr studied theoretical physics at Copenhagen University and worked as a posdoc at London University and also as a visiting scientist at several universities and institutes in Europe, the USA and Japan. Department in 1991 as a Senior Researcher and was appointed Director of the Centre for Biological Sequences Analysis in 1993. He studied physics at Copenhagen University before joining the DTU's Department of Structural Properties of Materials in 1988 as a Research Associate. He was awarded a PhD from the DTU in 1990 for work on system in genes. folding of proteins is therefore a common cause of biological failure. So it is vital to understand the basic features of protein folding, and to obtain some insight as to the material characteristics which are responsible for the unique structures that arise from the process.

We shall describe some of the intriguing aspects of protein folding and outline several puzzles that contemporary theories are unable to resolve. The purpose is to generate some debate on how to think about the protein folding problem as well as to suggest some possible different lines of thought. In particular, we shall suggest an alternative model which is able to account for some of these puzzles including, for example, the observation that some proteins consume heat on being transformed to a folded structure at low temperatures as well as on being transformed back to a disordered structure at high temperatures. The model is based on long-range excitations of the protein backbone that have not been considered before.

\section{The Main Puzzles}

\section{Cold denaturation}

The properties of proteins depend on the nature of the solvent. When proteins in an aqueous medium are heated, they loose their native three-dimensional structure and form a more random arrangement. This phase transformation is called denaturation and the transition temperature depends on the solvent. Surprisingly, upon cooling from their native state, many proteins (e.g., the protein beta-lactoglobulin) undergo an apparent re-entrance transition to the denatured state [1]. This process is called cold denaturation in contrast to the more commonly known phenomena of hot denaturation described above. It is a peculiar fact that when proteins are denaturated at high temperature they absorb heat, but when denaturated at low temperature they release heat. Contemporary theories describe protein folding as either driven by a decrease in entropy (as in spin-glass theories [2]) or by an advantageous gain in energetic interactions (as for a hydrophobic collapse [3]). The presence of the cold-denatured phase is unaccounted for in both cases. Cold denaturation is therefore a puzzle if protein folding is viewed as a balance of entropy and energy in its simplest context.

\section{Missing knots}

Strictly speaking, a knot is not topologically well defined for an open string (a string that has ends). However, most scientists would agree on what they would define as a knot in a protein, as well as on what they would define as a knot on a shoelace. Protein structures generally do not contain knots. In view of the often stated opinion that the long protein chains effectively search randomly through phase space, it appears puzzling that knots are not observed from time to time. This points to the existence of a mechanism which suppresses the formation of knots. The most well-studied theories of protein folding do not include such a mechanism as a generic property of protein folding. Some theories of the spin-glass type even allow relaxation that leads to a violation of the knot topology.

\section{Symmetry puzzle}

Many proteins display surprisingly beautiful symmetries $[3,4]$. Examples are: the four-bundle structure found in proteins such as haemerythrin, the Greek key beta-barrel structure found in proteins such as gamma-crystallin, and the alternating alpha-, beta-structure of the protein carboxypeptidase. In each case, the symmetry is caused by an apparent repetition of sub-structures. Chemically, proteins are composed of a chain of amino acids. It is therefore puzzling why the linear sequences of amino acids have so far failed to reveal the symmetry of the threedimensional protein structure formed by the polypeptide chain with the specific amino acid sequence. 


\section{The Levinthal Paradox}

Owing to the aperiodic nature of the amino acid sequence and to the relatively large number of common amino acids (twenty) with non-identical side-chains, the total number of non-equivalent structures, or configurations, of a typical protein is astronomically high. For large proteins, the total number of possible configurations can be written as $A^{N}$, where $A$ is the average number of conformations per amino acid and $N$ the number of amino acids. The time it takes to convert one conformation into another depends on the spatial difference between two conformations, but must be at least of the order of the time scale of molecular motions. Interatomic vibrations have typical values of $10^{-13} \mathrm{~s}$, and set a lower bound on the switching time between conformations. If $A$ is taken to be on the order of 10 and $N$ on the order of 125 (corresponding to a normal protein domain) we see that it would take on the order of $10^{112} \mathrm{~s}$ to scan all conformations.

Even in the context of astronomy, the time would be unrealistically large. This is what is generally referred to as the Levinthal paradox. One learns from the paradox that entropy reduction in the sense discussed above is not the principal mechanism behind protein folding: the path from unfolded to folded structures must instead be guided very strongly. Currently, theories based on so-called biased folding pathways are being studied [5] to resolve the paradox.

\section{Spin-glass theory}

The reason that random interaction spin-glass models have been popular for studying canonical features of the protein folding problem is the apparent irregularity and non-periodicity of the amino-acid sequence which makes up the polypeptide chain of a protein. A model which attempts to describe the transition of a protein from the approximately random structure of the one-dimensional chain to the correctly folded three-dimensional structure may therefore resemble the random-field model with its frustrated dynamics and many local minima. This model is described by a Hamiltonian containing self-interaction terms:

$$
H=\sum_{j} \sum_{k} J_{j k} S_{j} S_{k},
$$

where $J_{j k}$ are the apparent random exchange constants, and $S_{j}$ and $S_{k}$ are state variables representing the instantaneous structure of the protein. This Hamiltonian is equivalent to the random-field Hamiltonian. One of the essential features of spin-glass models is the existence of a glass transition. Its temperature can be estimated from mean-field theory as being proportional to the root mean-square fluctuation of the energies corresponding to incorrectly folded structures in the glass state. Upon lowering the temperature, it becomes very difficult below the glass transition for the polypeptide chain of a protein to acquire the correctly folded structure [2].

\section{Protein excitations}

Excitations may represent an attractive possibility for the mechanism controlling protein folding. However, in the study of one-dimensional systems such as chain molecules, only short-range excitations have hitherto been considered. This is because in the presence of disorder excitations must be localized [6], a topological restriction that does not hold for the physics of two- and three-dimensional systems.

There is in fact a one-dimensional case where long-range excitations can exist despite the presence of disorder. It involves circular molecules embedded in three-dimensional space such as the one depicted schematically in Fig. 1. The reason that chain molecules of this type can support long-range excitations stems from a conservation law for topology which is expressed by the White theorem stating that the linking number $L$ is a conserved integer [7] such that $L=W+T$, where $W$ is the writhing number and $T$ is the total twist of the backbone. In general terms, writhing expresses the path of a curve relative to itself and is given by the equation

$$
W=(1 / 4 \pi) \iint_{C \times c} \frac{\partial e}{\partial s_{1}} \times \frac{\partial e}{\partial s_{2}} \cdot e \cdot \mathrm{d} s_{1} \mathrm{~d} s_{2}
$$

where $e=\left(r_{2}-r_{1}\right) /\left|\left(r_{2}-r_{1}\right)\right|$. The vectors $r_{1}$ and $r_{2}$ describe the curve, and $s_{1}$ and $s_{2}$ are the distances along the curve. As can be seen from the White theorem, writhing can arise at the expense of twist. This is what is commonly observed in many domestic situations when twisted telephone cords release their twist by forming a double twist (a writhe). Even if the path of a closed curve locally resembles a random walk, the White theorem must be obeyed and forms the basis for correlations with a longer range than those for the local short-range order (see Fig. 1c).

Proteins are not closed curves. However, it is also too simple to view them as free strings in a vacuum. The reason for this is

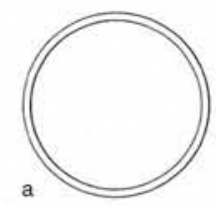

Fig. 1. Schematic representation of three configurations for a circular chain molecule in which the linking number is a conserved integer. a: An exactly circular configuration. b: Same as a) but with two opposite loops. c: Same as a) but with random short-range wriggles. The paths of a), b) and c) can continuously transform into each other.

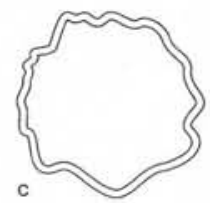

that proteins interact with an aqueous environment, and these interactions cannot be ignored, particularly not for short time scales. Similarly, when winding a hose onto a reel in the garden, gravity and the interaction of the hose with the lawn can become irritating hindrances, preventing the process from being completed on the shortest time scale. Large motions of the backbone of an unfolded protein in a aqueous solution take time, typically $10^{-5}-10^{-6} \mathrm{~s}$. On shorter time scales, the White theorem must be obeyed and the protein is subject to an effective topological constraint, as illustrated in Fig. 2.

\section{Wringons}

The effective topological constraint of polypeptide chains is the origin of longrange excitations of the chains with a range longer than the apparent local disorder. In the following we shall consider the polypeptide chain in the continuum limit where vibrational and torsional modes are separated. In a more realistic model composed of atoms and chemical bonds, a complete separation of vibrational and torsional motion will generally not be obtained. Nevertheless, some eigenmodes will still dominate the torsional behaviour. The vibrational modes correspond to phonons and the torsional modes we shall call wringons [8]. Wringons couple to the aqueous environment of the protein mainly through the induction of shear, while vibrational modes cause shear as well as compression. The order of the magnitude of the eigenfrequency for wring modes around the backbone can be estimated as approximately

$$
v=\frac{1}{2 \pi D}(y / i)^{1 / 2}
$$


where $D$ is the length of the backbone, $y$ the torsion constant per inverse length, and $i$ the moment of inertia of the backbone per unit length. Using values typical for molecular chains and bonds, the estimated frequency falls in the range $10^{8}-10^{10}$ $\mathrm{Hz}$. The periods of wring modes are therefore short enough to be consistent with the assumption that the protein is effectively topologically bound. Furthermore, a wring state of a polypeptide chain must be stable against the formation of curvature. The condition for this is found by comparing the potential energy of the torsion and curvature terms,

$$
E_{\text {pot }}=\frac{1}{2} \int\left[k_{\tau} \tau^{2}(l)+k_{\kappa} \kappa^{2}(l)\right] \mathrm{d} l
$$

where $k_{\mathrm{r}}$ and $k_{\mathrm{x}}$ are the effective elastic constants for twisting and for curving, respectively, per unit length at the position along the backbone characterised by the parameter $l$. The condition that a twisted line is stable against the formation of a helix (curvature), illustrated in Fig. 3 , is:

$$
2 k_{\mathrm{r}}<k_{\mathrm{\kappa}}
$$

The elastic properties of a polypeptide chain are non-linear and depend on the local chemical composition of the chain. The effective constants $k_{\mathrm{\tau}}$ and $k_{\mathrm{r}}$ may therefore obey the inequality at low amplitudes of the wring modes, while at larger amplitudes this is not so. At large amplitudes, the wring state becomes unstable and curvature is introduced. We suggest that this is the underlying mechanism for the initiation of protein folding [8]. The initiation process can be thought of as analogous to the famous collapse of the Tacoma Narrows Bridge in the State of Washington, USA, in 1940, when severe twist-like oscillations over the span of the bridge eventually lead to transverse fracturing of the bridge. Unlike proteins, the bridge did not have the option of forming a folded structure.

\section{Three phases}

Three situations arise in proteins: I) The inequality (1) is violated at all wring amplitudes. In this case, all wring modes are damped out;
II) The inequality (1) is valid at low wring amplitudes, but violated at higher wring amplitudes. In this case, a phase transformation can be initiated by wring modes leading to the folding of proteins; III) The inequality (1) is valid at all wring amplitudes. In this case, curvature is not formed. On the contrary, there is a ten-
Fig. 2. A schematic illustration of the region in time where chain molecules are subject to an effective topological constraint. Large motions of the polypeptide backbone can only arise at inverse times below $10^{5}-10^{6} \mathrm{~s}^{-1}$ so the protein is subject to an effective topological constraint. The backbone can consequently support a long-range excitation (a wringon or wring state) with a frequency of $10^{8}-10^{10} \mathrm{~Hz}$.

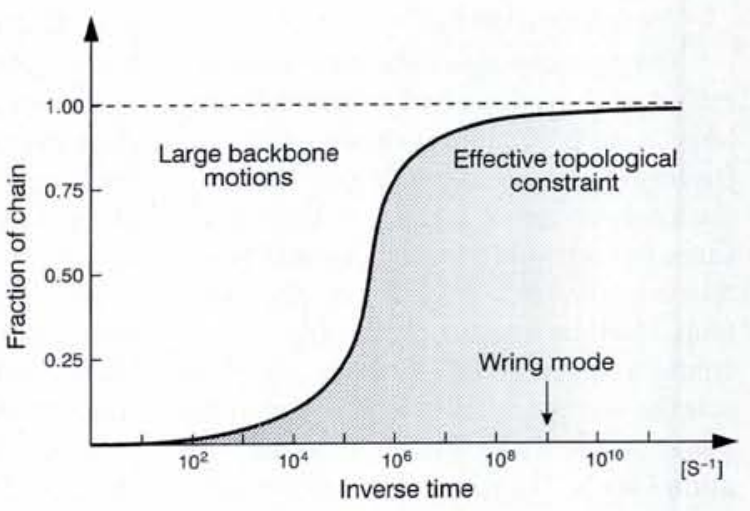

dency to reduce the amount of curvature, which leads to spontaneous denaturation.

These cases are consistent with the phase diagram commonly observed for proteins. For example, Fig. 4 shows the experimentally determined phase diagram for beta-lactoglobulin. It reveals three phases depending on the temperature and $\mathrm{pH}$, where I corresponds to the colddenaturated protein, II to the folded protein, and III to the hot-denaturated protein. The observations that heat is consumed during the transitions from both the cold-denaturated phase to the folded phase and from the folded phase to the hot-denaturated phase can be understood in terms of the wring model since the two transitions involve an increased amount of activity of the wring state.

\section{Protein Structure}

The large amount of information we have about proteins stems from the current very rapid growth of empirical data on the structure and functions of proteins. Since the determination by X-ray crystallography of the structure of heme-binding proteins, over two thousand different protein structures have been determined. Protein structures are identified using techniques such as X-ray and neutron diffraction, small-angle scattering, nuclear magnetic resonance, etc. The recently developed synchrotron-based X-ray methods constitute some very significant tools for structure determination. The threedimensional atomic coordinates for many of the proteins studied are available from

Fig. 3. A schematic illustration of a table-top model made of two hollow rubber tubes representing the two situations $2 k_{\tau}>k_{\mathrm{k}}$ (left-hand side tube) and $2 k_{\tau}<k_{\mathrm{k}}$ (right-hand side tube) corresponding to the condition that a twisted line is stable against the formation of a helical line. If the protein (tube) bends more easily than it twists, it bends and forms a helix; if it twists more easily than it bends it simply twists. the Brookhaven Protein Data Bank. Information about other biomolecular structures (e.g., DNA/RNA structures and antibody-antigen complexes) has recently been included.

One of the results that was obtained early on in the characterization of protein structures was the prediction and confirmation of forms of the polypeptide backbone common to otherwise very different proteins with different amino acid sequences. Such structures are referred to as secondary structure elements (see cover), by far the most abundant being the alpha-helix (a helical structure with 3.6 amino acids per turn which is stabilized by local hydrogen bonds along the backbone) and the beta-strand (a fully extended segment of a polypeptide chain).

The overall features of the folding pathways of smaller proteins such as lysozyme [9] have been studied by isotope NMR experiments, stopped-flow circular dichroism, fluorescence, and electrospray ionization mass spectrometry. The results indicated that the protein folding of lysozyme occurs in distinct stages. The first stage is dominated by the formation of secondary structure elements; disulfide

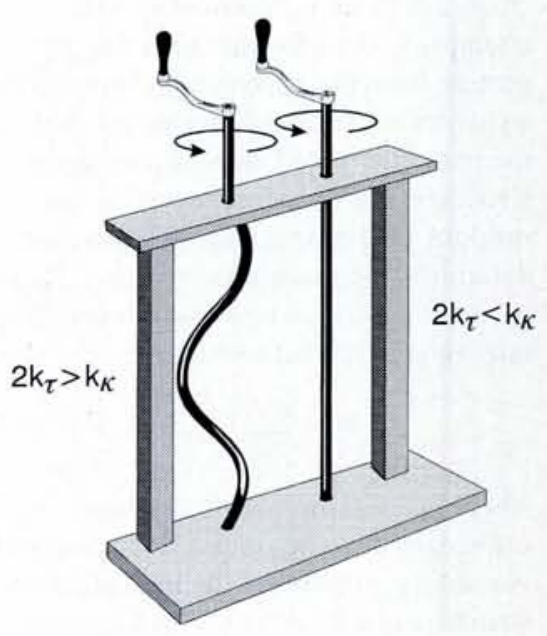


bridges are formed during the second stage, and the secondary structure elements are stabilized. At the third and final stage of the formation of the native state, single beta-strands join up to become double strands and the final docking of the secondary structure elements takes place. Recently, it has been shown that the folding of proteins can be very rapid. For the protein cytochrome-c, all the required intrinsic steps in its folding, including side-chain packing, are in fact inherently fast $(\approx 10 \mathrm{~ms})$ processes [10].

\section{Global wring modes}

In the wring model described above for protein folding, the resulting structure depends on the elastic constants of the polypeptide chain, which vary along the chain and depend on the local composition of the chain's amino acids. As the non-linearity of these constants is of great significance, the type and amplitude of the wring-mode pattern which is built up along the chain, and the way the amino acid sequence is aligned with the pattern, are crucial. Protein folding therefore involves both local and global aspects of the amino acid's sequence of proteins. Knowledge of the local aspects (the local elastic properties of the chain) are therefore insufficient for deciding which structure the chain will locally adapt. It is necessary, in addition, to have knowledge of the global collective wring modes.

\section{Stability of secondary structures}

If protein folding occurs in several distinct stages, in the sense that there is a tendency for secondary structure elements to be formed first, it is necessary that the secondary structures remain stable during the latter stages of protein folding. This is the situation for the alpha-helix. By contrast, a beta-strand is not stabilized by hydrogen bonds before an anti-parallel strand or a parallel double strand is formed. The feature that temporarily stabilizes a beta-strand is thus puzzling.

Within the scenario for protein folding put forward above, the secondary structures must be patterns that are formed early on and remain relatively stable under the influence of wring modes. Stability of this type can arise for backbone structures where the elastic constants are so non-linear that the mechanical properties of a protein's backbone become relatively independent of the specificity of the amino acids. This is the case for the alpha-helix, which is stabilized by local backbone hydrogen bonds.

Fig. 4. Phase diagram for beta-lactoglobulin in an acidic 4-molar urea solution. Three phases (cold-denaturated, folded protein, hotdenaturated) are present. The data points were determined by measuring the optical rotation in refolding experiments. Note that the transition temperature shifts to higher temperatures on increasing the $\mathrm{pH}$ for both transitions.

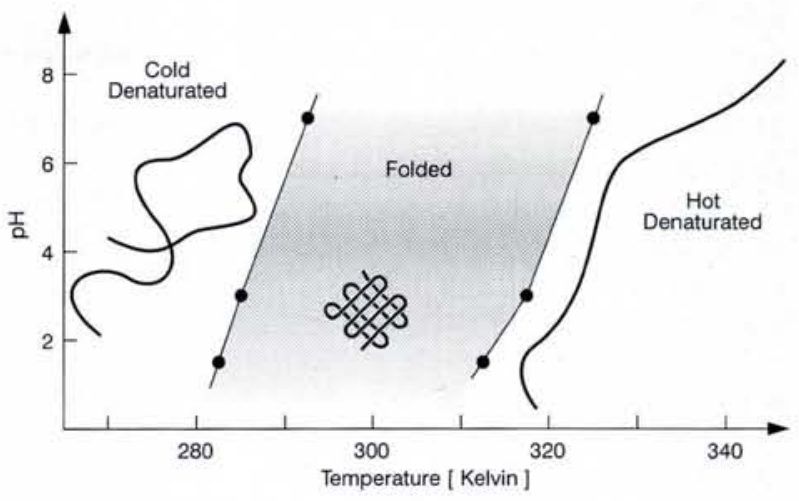

The other possibility for structural stability in the presence of wring modes is that $2 k_{\mathrm{r}}$ is less than $k_{\mathrm{k}}$ in a segment of the polypeptide chain. In this case, the wring modes will tend to dynamically stabilize the formation of extended polypeptide chains over more curved ones. A betastrand is an extended segment of a polypeptide chain, and it is therefore possible that beta-strands are stabilized by dynamic effects.

The validity of this hypothesis, namely, that beta-strands may be stabilized in their extended form by their dynamics, can be investigated by studying the hot-denaturated phase. As more of the protein obeys the inequality $2 k_{\mathrm{\tau}}<k_{\kappa}$, the hot-denaturated protein should contain a number of single beta-strands. Moreover, the existence of proteins that undergo cold denaturation points to the intriguing possibility that in some cases the presence of wring modes is important for the stability of a protein structure.

\section{Outlook}

One of the interesting predictions of the wring-mode description is that colddenatured and hot-denatured proteins are thermodynamically distinguishable - a view that is not shared by many theorists. However, it is one which is consistent with the experimentally determined phase diagram for beta-lactoglobulin presented above.

\section{Protein length}

The model is by its nature dependent on the length of the polypeptide chain, since a change in the length would also alter the standing wring modes (eigenmodes). This prediction can be put to the test in specially designed experiments using modified proteins. First, it implies that proteins of different lengths can fold in different ways. Second, as the frequency of the eigenmode depends on the length, it can also mean that the resonance condition necessary for obtaining wring-mode activity of sufficient amplitude is not obtained at all lengths, but only at favourable lengths [8]. This points to the possibility that proteins of all lengths are not equally common. In fact, there is statistical evidence that this is the case: a typical one-domain protein is 125 amino acids long [11]. Of course, one may argue that many proteins have not yet been studied or discovered. The preference for specific lengths can also been seen empirically in multi-domain proteins. In proteins of this type, all domains are about the same size.

Direct confirmation of the existence of wring modes using spectroscopy would be an important test of the model. Microwave spectra reveal features in the correct frequency range [12] - features that have been linked to the presence of water bound to the protein molecule, for when proteins are dehydrated the microwave absorption disappears. It is necessary to consider two alternative possibilities: a) that the disappearance of the microwave absorption is directly related to the lack of bound water molecules; b) that the disappearance is due to a change in the nature of the topological constraint of the protein molecule, i.e., the dehydrated protein is not topologically constrained at the wring mode frequency.

\section{Communication}

There are other, major unresolved questions concerning the physics of proteins, notably the mechanism by which protein-protein, peptide-protein, or ligand-protein interactions can lead to communication in biological systems. By communication we mean that one molecular event can start one or more other events. The standard model is that the affinity of a peptide-protein docking can be very specific; i.e., the docking only takes place if the "fit" is perfect. This explains the specificity of proteins and 
peptides. However, it contradicts the fact that one side of a protein knows what has happened on the other side - a property which is needed for there to be communication across a protein.

When dynamical effects such as collective long-range modes are included in the description of proteins, it is clear that the docking of a peptide at a specific site will have a global influence on the wring mode - the standing collective mode of the folded protein (type II for the inequality above). Long-range wring modes can thereby form the foundation for communication. The importance of wring modes for protein-protein communication and peptide-protein chemistry will therefore need to be considered.

The existence of proteins with many sub-conformations has been suggested [13]. We note that switching between different wring states provides a means to switch between the different states of a protein.

\section{Enzymatic and electromagnetic activities}

The mechanism whereby some proteins exhibit enzymatic activity is not fully understood. Standing wring modes in proteins can provide an attractive energy basin as well as mechanical action in this regard. Finally, there appears to be evidence that the interaction of non-ionizing electromagnetic radiation with biological systems is not completely understood, as is suggested by recent epidemiological studies [14]. Collective modes in proteins and other chain molecules will couple to electromagnetic radiation and therefore form the basis for an additional interaction between electromagnetic radiation and biological systems.

\section{References}

[1] Griko Y.V., et al., J. Mol. Biol. 202 (1988)127. [2] Wolynes P.G., Onuchic J.N. \& Thirumalai D., Science 276 (1995) 1619.

[3] Dill K.A., et al., Protein Sci. 4 (1995) 561. [4] Branden C. \& Tooze J., Inroduction to Protein Structure (Garland, NY) 1991.

[5] Zwanzig R., Proc. Nat. Acad. Sci. USA 92 (1995) 9801.

[6] Iori G., Int. J. Neural Systems 3 (1992) 201.

[7] White J.H., Amer. J. Math. 91 (1969) 693.

[8] Bohr J., Bohr H. \& Brunak S., in: Protein Folds: A Distance-Based Approach; Eds. H.

Bohr \& S. Brunak (CRC Press, New York) 1995. [9] Eyles S.J., et al., Biochem. 33 (1994) 13038. [10] Sosnick T.R., et al., Biophys. J. 70 (1996) A 378 .

[11] Berman A., et al., Proc. Natl. Acad. Sci. USA 91 (1994) 4044.

[12] Miura N., et al., Biopolymers 34 (1994) 357. [13] Frauenfelder H., Sidlar S.G. \& Wolynes P.G., Science 254 (1991) 1598.

[14] Infante-Rivard C., The Lancet 346 (1995) 177.

\section{Highlights}

\section{Element 112 Discovered}

An international team based at the Gesellschaft für Schwerionenforschung (GSI), Darmstadt, and led for more than 20 years by P. Armbruster discovered on 9 February 1996 its sixth element, which has an atomic number of 112 and is the heaviest ever produced [S. Hofmann, et al., submitted to Z. Physik A, 21 February 1996]. Two decay chains of the isotope ${ }^{277} 112$ were observed in irradiations of ${ }^{208} \mathrm{~Pb}$ targets with ${ }^{\circ} \mathrm{Zn}$ projectiles having a kinetic energy of $344 \mathrm{MeV}$. The isotope decayed by the emission of $\alpha$-particles with a half-life of $240_{-90}^{+430} \mathrm{~ms}$. Two different $\alpha$-energies were measured for the two observed decays.

Previous work [see EN 26 (1995) 103] using ${ }^{208} \mathrm{~Pb}$ and ${ }^{204} \mathrm{Bi}$ had led to the discovery of ${ }^{269} 111$, ${ }^{271} 110$ and ${ }^{272} 111$ nuclei produced by fusion reactions with the emission of one neutron. The measured cross-sections gave an estimated cross-section of $1-3 \mathrm{pb}$ for the production of the isotope ${ }^{277} 112$ by the ${ }^{70} \mathrm{Zn}+{ }^{208} \mathrm{~Pb}$ reaction (the measured value turned out to be $1.0_{-0.4}^{+1.2} \mathrm{pb}$ ).

The energy of the $\alpha$-particles allows a very important theoretical prediction to be verified. Atomic nuclei with 162 neutrons should be more strongly bound than their neighbours because of their inner structure. Such structure phenomena led to the prediction some 30 years ago that super-heavy nuclei with a neutron number near 184 could exist; the same structure explains the existence of the six heaviest elements, all of which have been discovered at GSI. The decay chain of element 112, which demonstrates the stabilization at 162 neutrons, confirms the predictive power of the model for nuclear structure and must be regarded as a major success for nuclear physics.

The team had looked for the isotope ${ }^{290} 116$ late last year, but since systematics in this range of atomic numbers are less well established than for 112, identifying the optimum beam energy was difficult. Nonetheless, the successful production of the element 112 confirms that it should be possible to continue to penetrate further into the region of super-heavy atoms.

\section{Data Question Quark Structure}

The 450-member Collider Detector at Fermilab (CDF) collaboration has reported data [F. Abe, et al., FERMILAB-PUB-96/o20-E; submitted to Phys. Rev. Lett. (24 January 1996)] from experiments carried out over the past two years at the Fermi National Accelerator Laboratory's Tevatron Collider showing that the probability of particle scattering in high-energy proton-antiproton collisions is significantly larger than that predicted by current theoretical models. Collaborators believe that more studies of both experimental data and theoretical analyses will show whether relatively small adjustments to theory can reconcile the discrepancy, or whether the data are the first hint that quarks are made up of something smaller and may not be fundamental after all.

One prosaic explanation for the observed discrepancy is that the quark-gluon mixture

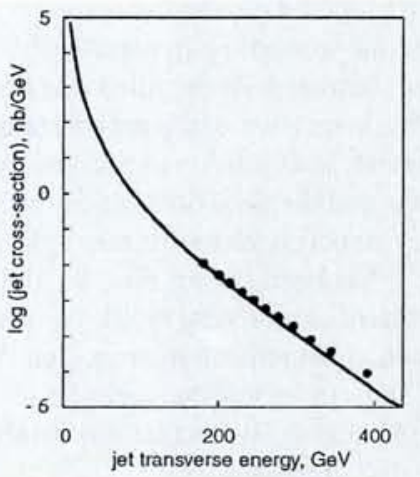

A comparison of the inclusive jet cross-section (points) for $\bar{p} p$ collisions observed at CDF with the cross-section predicted by perturbative quantum chromodynamics for jet transverse energies of $15-440 \mathrm{GeV}$. The CDF cross-section is larger than the $Q C D$ prediction above $200 \mathrm{GeV}$. From F. Abe, et al., FERMILAB-PUB-96/020-E.

which makes up the proton shares the momentum of the proton in such a way that one piece carries a larger fraction of the momentum than previously believed. This interpretation needs to be reconciled with a large body of data from many other experiments.

\section{A Dry, Windy, Rocky Jupiter}

Preliminary analyses of data from the Galileo atmospheric probe that plunged into Jupiter last December gave an estimated ratio of helium to hydrogen by mass - a key parameter for theories of planetary evolution - of only $14 \%$ for Jupiter. A more comprehensive analysis has raised this estimate to $24 \%$, so the ratio of the two elements making up $99 \%$ of the Jovian atmosphere closely matches that found in the Sun. This suggests that Jupiter's bulk composition has not changed since the planet formed from the primitive solar nebula which spawned the planets. The revised helium abundance also indicates that gravitational settling of helium toward the interior of Jupiter has not occurred nearly as fast as it apparently has on Saturn, where the approximate helium-tohydrogen ratio is $6 \%$. This may force a revision of projections for the size of the rocky core believed to exist deep in the centre of Jupiter.

Estimated amounts of key heavy elements such as carbon and sulphur (probably from the influx of meteorites and other small bodies) have increased, but minimal organic compounds were detected. The detection of minimal amounts of organic compounds indicate that such complex combinations of carbon and hydrogen are rare on Jupiter, and that the chances of finding biological activity are extremely remote. Measurements also suggest much less water than the amounts found on the Sun and predicted from data sent by the Voyager spacecraft that flew by Jupiter in 1979.

Preliminary estimates of wind speeds have grown from $530 \mathrm{~km} / \mathrm{h}$ to more than $640 \mathrm{~km} / \mathrm{h}$. The winds persisted far below the one cloud layer detected, strongly suggesting that the winds - a feature of all the giant outer planets are driven by heat escaping from deep in the planet's interior rather than by solar heating. 